\title{
CONSTRUCTIONS OF ASTROMETRIC INSTRUMENTATION AT PULKOVO OBSERVATORY
}

\author{
V.N.YERSHOV ${ }^{1}$, YU.S.STRELETSKY ${ }^{1}$ AND N.V.LEBEDEV ${ }^{2}$ \\ 1 Central Astronomical Observatory USSR Academy of Sciences \\ Pulkovo \\ 196140 Leningrad \\ USSR \\ ${ }^{2}$ Television Institute \\ Leningrad, USSR
}

ABSTRACT. Past and present construction of astrometric instrumentation at Pulkovo is discussed.

From the very beginning of Pulkovo Observatory, special attention was paid by V.Ya. Struve to the construction of astrometric instrumentation. First, astrometric instruments ordered from Germany (such as the Vertical Circle and the Large Transit Instrument) were constructed under his close participation. The tradition of the participation of leading scientists in the production of astrometric instruments remains up to the present day.

Many fine instruments were created within the facilities of Pulkovo Observatory. For example, in 1904, the talented Pulkovo mechanic G.A.Freyberg fabricated a zenith-telescope ZTF-135 which has not lost its value as a scientific instrument even today. In the 1930s, N.N. Pavlov successfully used a method of photoelectric registration for transit instruments, thereby improving the time determination accuracy to $0.01 \mathrm{~s}$.

In the 1950 s and 1960 s some new astrometric instruments were created, such as the thermally insulated transit instrument of N.N. Pavlov, A.A.Mikhailov's photographic vertical circle, and the large transit instrument for expeditions. Following the design of V.P. Linnik, the 6-meter baseline optical interferometer was created for the measurement of double stars. D.D. Maksutov, N.N. Mikhelson $e t$ al. designed at Pulkovo the fine two-meniscus wide field AZT-16 astrograph. During this period, Pulkovo astrometrists participated in the design process of the zenith-telescope ZTL-180 (V.I. Sakharov, I.F. Korbut) and of the photographic zenith tube (A.A. Mikhailov and V.A. Naumov).

The new instruments allowed the achievement of higher accuracy in stellar coodinates, latitude, longitude and time determination. Three instruments (AZT-16, the photographic vertical circle and the large transit instrument) were used in the Chile expedition.

In the 1970s and 1980s, the work of reconstruction and modernization of old instruments was carried out at Pulkovo. The design of the photographic vertical circle was essentially improved by B.K. Bagildinsky and V.D. Schkutov. The horizontal meridian circle in this period was fully automated. A scanning micrometer and a new photoelectric circle reading system were installed. 
The modernization was carried out by G.I. Pinigin, T.R. Kiryan, R.I. Gumerov and other contributors of the Pulkovo and Engelhardt observatories.

The Toepfer meridian circle was used for a new semi- automatic instrument named MK-200. A scanning photoelectric micrometer was first installed on it and linear CCD's were used for the circle reading micrometers. The reconstruction of the Toepfer was done by Yu.S. Streletsky, Yu.G. Ostrensky, V.N. Yershov and others. Significant works on astrometric instrumentation were implemented at the Nikolayev department of Pulkovo Observatory. In the 1960s, a new circle reading device for the Repsold meridian circle was created and a circle division laboratory was formed. In 1980, the Repsold meridian circle was equipped with a photo-electric micrometer, giving an accuracy of 0.28. This was done by V.V. Konin, L.G. Karyakina, A.D. Pogony and others.

In this observatory, prototype of G.I. Pinigin's and O.E. Shornikov's axial meridian circle was designed and produced and the automatic measuring machine was made for astroplates (IFO-461). The main contributions to this work were made by A.V. Sergeev and O.E. Shornikov.

It is very urgent now to replace mechanical micrometers in meridian instruments by fixed micrometers based on the CCD. Accuracy of this micrometer increases at the cost of hard geometrical features and of obtaining more information about stellar images. With cooling and using a signal accumulation time of about $30 \mathrm{~s}$, one can expand the magnitude limit of a standard meridian circle down to14-17 magnitude. This gives a possibility to solve new tasks by the means of the classical meridian method (e.g., the connection of the optical reference frame to the inertial VLBI coordinate system). Additional efficiency can be obtained at the cost of increasing the variety of observing objects, such as close binary stars. New possibilities are opened when observing day-time objects (Venus, Mercury, and so on).

Some experiments were made in 1988 with CCD observations with the MK-200 meridian circle. This had a magnitude limit of about 8.8. In 1989, some trial observations with a complex CCD micrometer were made in collaboration with the Leningrad Television Institute. The micrometer can automatically change its working mode and parameters. As a model of a meridian circle, a small telescope $(\mathrm{D}=11 \mathrm{~cm}, \mathrm{f}=80 \mathrm{~cm})$ was used. The estimation of accuracy made on the basis of $\alpha O p h$ observations gives a value 0." 3 per $10 \mathrm{~ms}$. During day-time observations of Polaris, the signal to noise ratio was about 50 , which gives a day-time magnitude limit of about 4.5 .

Now some new astrometric instruments are being designed at Pulkovo Observatory. And following the tradition at Pulkovo, astrometrists participate directly in the creation of the instruments.

On the basis of experience with Sukharev's horizontal meridian circle investigations, a new automatic horizontal meridian circle (SMAHI) is being developed, which could give systematic errors less then 0."05. The organizer of the work is G.I. Pinigin.

Based on proposals of A.A. Nemiro and Yu.S. Streletsky, a reflecting automatic meridian circle is under construction. This instrument will give an improved limiting magnitude. Optical elements of the meridian circle will be checked by the special autocollimating systems and therefore the systematic errors will be minimized. A design of the photoelectric zenith tube is completed and is being implemented now. This instrument has an image dissector tube as a photodetector. As a prospect to the future, space astrometric telescopes are under consideration at Pulkovo Observatory. 